OPEN ACCESS

Edited by: Annalisa Lonetti, University of Bologna, Italy

Reviewed by:

Yona Keisari,

Tel Aviv University, Israel Obul Reddy Bandapalli, Hopp Children's Cancer Center

Heidelberg (KiTZ), Germany

${ }^{*}$ Correspondence:

Jixian Luo

luojx@sxu.edu.cn

Specialty section:

This article was submitted to Hematologic Malignancies, a section of the journal Frontiers in Oncology

Received: 03 April 2020

Accepted: 14 July 2020

Published: 07 August 2020

Citation:

Luo J, Wang J, Zheng $\mathrm{H}$ and Wang L (2020) Rho GDP-Dissociation Inhibitor 2 Inhibits C-X-C Chemokine Receptor Type 4-Mediated Acute Lymphoblastic Leukemia Cell Migration. Front. Oncol. 10:1512. doi: 10.3389/fonc.2020.01512

\section{Rho GDP-Dissociation Inhibitor 2 Inhibits C-X-C Chemokine Receptor Type 4-Mediated Acute Lymphoblastic Leukemia Cell Migration}

\author{
Jixian Luo*, Junting Wang, Huiguang Zheng and Lan Wang \\ School of Life Sciences, Shanxi University, Taiyuan, China
}

Although we currently have a good understanding of the role $\mathrm{C}-\mathrm{X}-\mathrm{C}$ chemokine receptor type 4 (CXCR4) plays in T cell acute lymphoblastic leukemia (T-ALL), the mechanism of CXCR4-mediated T-ALL migration remains elusive. Therefore, we focus on the downstream signals of CXCR4 that contribute to T-ALL cell migration in this study. Rho GDP-dissociation inhibitor 2 (RhoGDI2) is expressed preferentially in lymphocytes. It interacts with and regulates the activation of Rho proteins by inhibiting the dissociation of GDP and the binding of GTP. In a previous study, we demonstrated that RhoA and RhoC are activated and required for CXCR4-mediated JURKAT cell migration. In the present work, we investigate the role of RhoGDI2 in CXCR4-mediated T-ALL cell migration. Results show that RhoGDI2 sh2 significantly releases its inhibition effects on T-ALL cell migration toward CXCL12 (C-X-C motif chemokine ligand 12). Phosphorylation of RhoGDI2 on Y24 and Y153 releases RhoA and RhoC from RhoGDI2, which recovers CXCR4-mediated migration toward CXCL12 although the phosphorylation of Y130 has less effect on RhoA or RhoC binding. Furthermore, Src is activated by CXCL12. Transfection of siRNAs to Src reduces CXCR4-mediated migration. Src is required for the phosphorylation of RhoGDI2 on Y153, and ABL1 is activated by CXCL12 and responsible for the phosphorylation of RhoGDI2 on Y24 and Y130. Similarly, knockdown of the expression of ABL1 by siRNAs reduces the CXCR4-mediated migration. Therefore, RhoGDI2 may be a brake for CXCR4-positive T-ALL migration. Because migration is a prerequisite for infiltration of leukemia, this work may suggest the possible involvement of RhoGDI2 in infiltration of T-ALL.

Keywords: RhoGTPases, migration, leukemia, chemokines, receptor

\section{INTRODUCTION}

Acute lymphoblastic leukemia (ALL) accounts for $70-80 \%$ of all leukemia (1). Although the development of clinical treatments have resulted in a cure rate of about $85-90 \%$ (2), relapses occur in about $15-20 \%$ of children and are still the major reason for early age mortality (3). CXCR4-mediated infiltration of the central nervous system is a severe trait of $\mathrm{T}$ cell acute 
lymphoblastic leukemia (T-ALL) and correlates with relapses (4). Accumulating data have demonstrated that high expression of CXCR4 is associated with infiltration into spleen, liver, lymph nodes, and CNS in T-ALL (4-8). In the critical process of infiltration, transendothelial migration of leukemic cells is required to exit the blood stream into target organs, which is the prerequisite for infiltration (9). Therefore, the mechanism of CXCR4-mediated migration of T-ALL cells is focused on in the following study.

Rho GDP-dissociation inhibitor 2, RhoGDI2, alternative name Ly-GDI or Rho-GDI beta, is expressed preferentially in lymphocytes (10). It interacts with the Rho proteins, including RhoA (11), Cdc42 or Rac1 (12), and Rac2 (13), and regulates the activation of Rho proteins by inhibiting the dissociation of GDP and the subsequent binding of GTP $(10,14)$. Therefore, it regulates reorganization of the actin cytoskeleton mediated by Rho family members (15). RhoGDI2 high expression has been found in ALL cell lines, for example, the JURKAT T-ALL cell line (16). However, the function of RhoGDI2 in ALL migration remains largely unknown. In this paper, we design experiments to investigate the role of RhoGDI2 in CXCR4-mediated T-ALL cell migration.

\section{MATERIALS AND METHODS}

\section{Cell Lines}

JURKAT (Clone E6-1) and CCRF-CEM cell lines were kindly provided by Stem Cell Bank, Chinese Academy of Sciences. HEK293T cells were purchased from the American Type Culture Collection (ATCC).

\section{Reagents or Antibodies}

AMD3100 (S8030, Plerixafor, antagonist against CXCR4) was purchased from Selleckchem. Poly-D-lysine (C0312) was purchased from Beyotime Biotechnology. ATP (disodium salt) (IA0590) was purchased from Solarbio.

Recombinant human Src protein (ab79635, His tag active Src) was purchased from Abcam. Recombinant human/feline/rhesus macaque (350-NS) was purchased from R\&D systems.

Antibody to human CXCR4 (ab181020) and anti-GFP antibody (ab6556) were purchased from Abcam. Antibodies to RhoGDI2 (sc-271042) and ABL1 (sc-56887) were purchased from Santa Cruz biotechnology. Anti-RhoGDI2 (D262966) for Western blot assay, anti-ACTB (D110001), anti-Src antibody

TABLE 1 | siRNA sequences or primers designed to construct the corresponding plasmids.

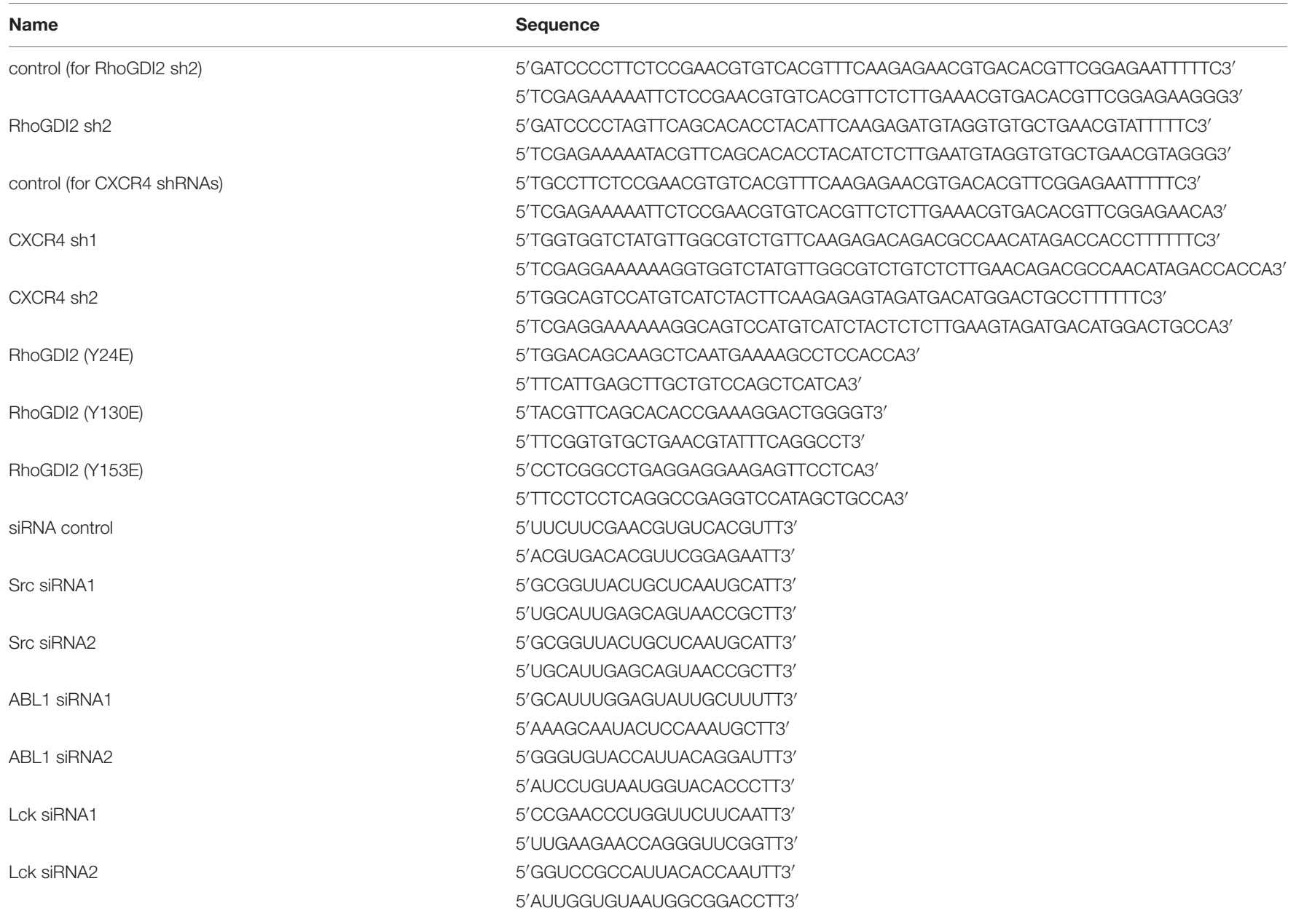


(D221267), and anti-GST-Tag pAb (AE006) were purchased from Sangon Biotech Company. Rabbit $\mathrm{mAb}$ to phospho-Src family (Tyr416) (D49G4), rabbit $\mathrm{mAb}$ to RhoA (67B9), and rabbit $\mathrm{mAb}$ to RhoC (D40E4) were purchased from Cell Signaling Technology. Rabbit mAb to phospho-Lck family (Tyr394) (D155064) was purchased Sangon Biotech. Mouse anti-HisTag mAb (AE003) was purchased from ABclonal. Monoclonal antibody to phosphotyrosine produced in mouse (PY20, P4110) was purchased from Sigma-Aldrich. Fast Mutagenesis System (K11209) was purchased from TransGen Biotech.

\section{rDNA Constructs}

To construct GST-fused Crk-CTD domain plasmids, CRKCTD was amplified by PCR using the cDNA from JURKAT cells as a template and cloned into the vector pGEX-4T-1. The open reading frame of the inserted DNA was confirmed by sequencing, and the expression of the GST-fused proteins was verified by SDS-PAGE. Plasmids expressing wild-type (WT) GST-tagged human RhoGDI2 and mutants (Y24F, Y130F, and Y153F) in pGEX-6p-1 vector, GFP-tagged WT human RhoGDI2 in lentiviral pWPXLd vector, and shRNAs targeting RhoGDI2 or random control in lentiviral pDSL_hpUGIP vector were kindly provided by Professor XL Zeng from Northeast Normal University, Changchun, China. Mutants expressing GSTtagged RhoGDI2 Y24E, Y130E, and Y153E and GFP-tagged RhoGDI2 Y24E, Y130E, and Y153E were amplified by PCR using point mutation kits of the Fast Mutagenesis System (K11209) from TransGen Biotech. In this PCR, WT RhoGDI2 plasmid was the template, and primers are shown in Table 1. Mutants expressing GST-tagged RhoGDI2 Y24E130E, RhoGDI2 Y24E153E, RhoGDI2 Y130E153E, and RhoGDI2 Y24E130E153E were amplified similarly by two or three rounds PCR using RhoGDI2 Y24E or Y130E as templates, and the mutants were verified with sequencing.

For CXCR4 shRNA preparation, annealed double-stranded shRNA oligonucleotides shown in Table 1 were cloned into the XhoI and HpaI cloning sites of lentiviral pLL3.7 vector with GFP expressing sequence.

\section{Generation of Lentiviruses and Transfection}

RhoGDI2 WT, Y24E, Y130E, and Y153E in pWPXLd and CXCR4 shRNAs in pLL3.7 or RhoGDI2 shRNA2 in pDSL_hpUGIP were transfected together with psPAX2 and pMD2 (at a ratio of 4:3:1) into HEK293T cells with lipofectamine 2000 (Invitrogen) to generate lentiviruses. Viral stocks were made to infect Jurkat cells or CCRFCEM cells. After $72 \mathrm{~h}$, cells were collected, and cell lysate was used in the following Western blot assay to detect interfering efficiency.

\section{Small Interfering RNA}

JURKAT or CCRF-CEM cells, $1.5 \times 10^{6}$, were seeded in each well of a 24 -well plate precoated with $0.1 \mathrm{mg} / \mathrm{ml}$ polyD-Lysine cultured in $500 \mu \mathrm{l}$ RPMI 1640 medium without antibiotics in a $37^{\circ} \mathrm{C}$ incubator containing $5 \% \mathrm{CO}_{2}$ for 20 $24 \mathrm{~h}$. Cells attached to poly-D-Lysine-coated wells were washed three times with PBS and then transiently transfected with small interfering RNAs (siRNAs) targeting $S r c, A B L 1$, or control siRNA using Lipofectamine 2000 following the manufacturer's instructions for adherent cells. Four to $6 \mathrm{~h}$ after transfection, cells were replaced with complete medium. SiRNAs were used at $200 \mathrm{pM}$. After $48 \mathrm{~h}$, cells were collected for the following Western blot assay to detect interfering efficiency or transwell assay.

\section{Transwell Assay (in vitro Migration Assay)}

The migration of JURKAT or CCRF-CEM cells was evaluated using a transwell chamber with $3-\mu \mathrm{m}$ pores (Corning). Cells were starved in medium containing $0.5 \%$ FBS overnight. Then $3 \times 10^{5}$ cells were seeded in the upper wells, and $25 \mathrm{ng} / \mathrm{ml} \mathrm{CXCL12}$ was placed in the lower wells. The plates were incubated at $37^{\circ} \mathrm{C}$ for $4 \mathrm{~h}$ with $5 \% \mathrm{CO}_{2}$. After each experiment, each upper well was gently taken away, and eight pictures of the cells that had migrated to the lower wells were taken randomly under a fourfold objective of a phase contrast microscope. Relative migration rate was the result of dividing the cell number of each group by that of the control group.

\section{GST Pull-Down Assay}

GST and GST-fused proteins (GST-CRK-CTD, GSTRhoGDI2-WT, GST-RhoGDI2-Y24E, GST-RhoGDI2 Y130E, GST-RhoGDI2 Y153E, GST-RhoGDI2 Y24E Y130E, GSTRhoGDI2 Y24E Y153E, GST-RhoGDI2 Y130E Y153E, GST-RhoGDI2 Y24E Y130E Y153E, GST-RhoGDI2 Y24F, GSTRhoGDI2 Y130F, and GST-RhoGDI2 Y153F) were expressed in Escherichia coli strain BL21. The induction was performed by adding $1 \mathrm{mM}$ isopropyl- $\beta$-d-thiogalactopyranoside (IPTG) to the culture with $\mathrm{OD} 1.0$ at $37^{\circ} \mathrm{C}$ for $3 \mathrm{~h}$. Whole bacteria lysates were applied to GST-BindTM Resin beads (D00172269, EMD Millipore Corp., MA, United States), and GST-tagged proteins were purified according to the manufacturer's instructions. For pull-down experiments, JURKAT and CCRF-CEM cells were starved in medium containing $0.5 \%$ FBS. Then, $4 \times 10^{7}$ cells were lysed in pull-down lysis buffer $(25 \mathrm{mM}$ Tris, $\mathrm{pH} 7.5,150 \mathrm{mM} \mathrm{NaCl}, 1 \%$ Non-idet P-40, 5\% glycerol, $60 \mathrm{mM} \mathrm{MgCl}_{2}$ ) with freshly added PMSF, and $30 \mu \mathrm{l}$ of each sample were taken for basal control. The left lysates were incubated with $25 \mu \mathrm{g}$ GST-tagged proteins bound to glutathione Sepharose $4 \mathrm{~B}$ beads at $4^{\circ} \mathrm{C}$ for $4 \mathrm{~h}$. After three washes with lysis buffer, the bound proteins were analyzed by Western blot assay.

\section{In vitro Kinase Assay}

Escherichia coli BL21 expressing GST-RhoGDI2-WT, GST-RhoGDI2-Y24F, GST-RhoGDI2-Y130F, or GSTRhoGDI2-Y153F were induced by $1 \mathrm{mM}$ IPTG, sonicated, and purified by using GST-BindTM resin beads according to the manufacturer's instructions. 
Next, 600 ng recombinant human active Src was coincubated with $100 \mu \mathrm{M}$ ATP, $1.2 \mu \mathrm{g}$ of GST-RhoGDI2 (WT or mutated) fusion protein in $40 \mu \mathrm{l}$ of kinase buffer $(5 \mathrm{mM}$ $\beta$-glycerophosphate, $25 \mathrm{mM}$ Tris, $\mathrm{pH}$ 7.5, $2 \mathrm{mM}$ DTT, $1 \mathrm{mM}$ $\mathrm{Na} 3 \mathrm{VO} 4,10 \mathrm{mM} \mathrm{MgCl}_{2}$ ) at $30^{\circ} \mathrm{C}$ for $30 \mathrm{~min}$. The reactions were terminated by boiling with $10 \mu \mathrm{l}$ of $5 \times$ Loading buffer
A

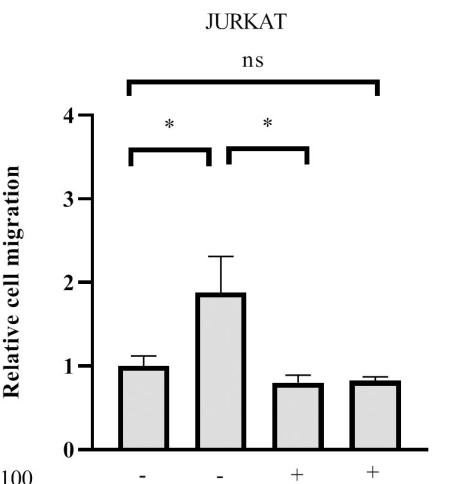

AMD3100

CXCL12

C

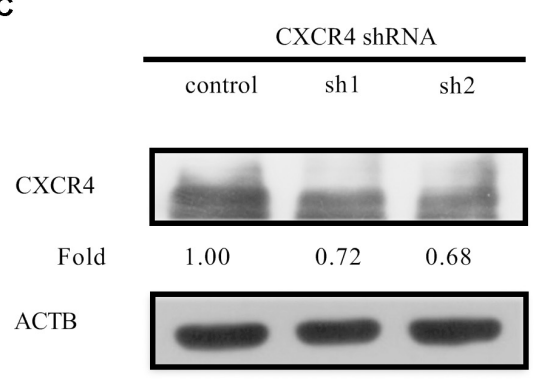

JURKAT

E JURKAT

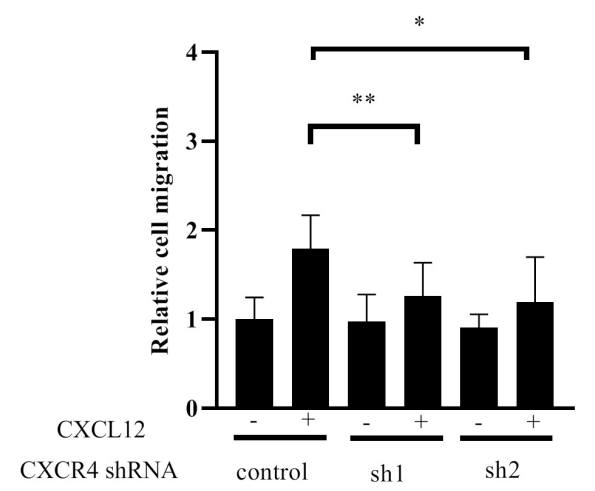

B

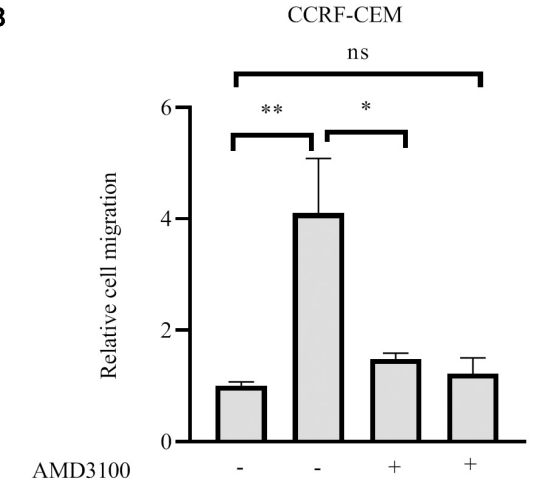

CXCL12

D

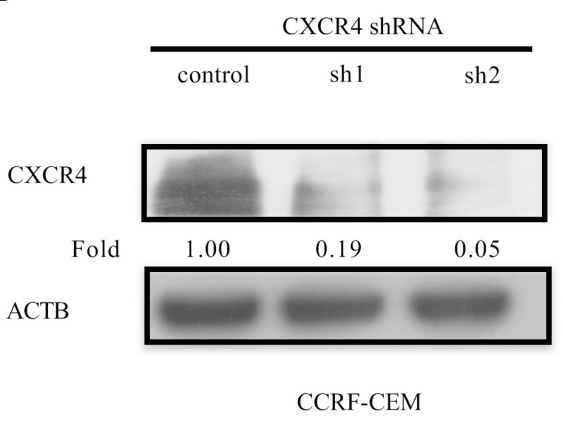

F CCRF-CEM

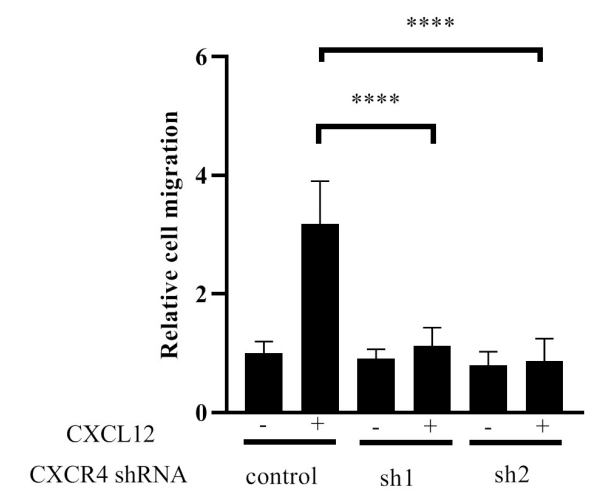

FIGURE 1 | CXCR4 mediates T-ALL cell migration toward CXCL12. (A,B) Starved cells were pretreated with $500 \mathrm{ng} / \mathrm{ml}$ AMD3100 or equal concentration of solvent on ice for $30 \mathrm{~min}$, washed with medium, and $3 \times 10^{5}$ cells in $100 \mu \mathrm{l}$ were used in the following transwell assay. The relative migration rate is shown. (C-F) Cells were infected with lentivirus containing CXCR4 shRNAs and control shRNA. Then, $72 \mathrm{~h}$ after infection, the interference efficiency of shRNAs to CXCR4 was detected by Western blot using CXCR4 antibody (C,D), and transwell assays were conducted (E,F). Each experiment was repeated at least three times. Western blot bands were analyzed using Image Pro Plus 6.0, and fold changes of CXCR4 were normalized according to ACTB. ACTB was used as the loading controls. ${ }^{*} p<0.05$, ${ }^{* \star} p<0.01,{ }^{* \star \star *} p<0.0001$. 
together at $98^{\circ} \mathrm{C}$ for $5 \mathrm{~min}$. Then samples were resolved by SDS-PAGE. CCRF-CEM cells were stimulated at $37^{\circ} \mathrm{C}$ with CXCL12 (25 ng/ml) for $30 \mathrm{~min}$, and active ABL1 were immunoprecipitated using ABL1 antibody as previously described (17). Immunoprecipitates were washed with kinase buffer and added to the kinase buffer containing containing $2 \mu \mathrm{g}$ of GST-RhoGDI2 mutated fusion protein.

\section{Western Blot Assay}

JURKAT or CCRF-CEM cells $\left(3 \times 10^{6}\right.$ per sample $)$ were stimulated as described above and lysed in lysis buffer $(50 \mathrm{mM}$ Tris, $\mathrm{pH}$ 7.5, $150 \mathrm{mM} \mathrm{NaCl}, 1 \mathrm{mM}$ EDTA, $1 \mathrm{mM}$ EGTA, $1 \%$ Non-idet P-40, $2.5 \mathrm{mM}$ sodium pyrophosphate, $1 \mathrm{mM}$ glycerophosphate, $1 \mathrm{mM} \mathrm{Na}_{3} \mathrm{VO}_{4}, 1 \mathrm{mM} \mathrm{NaF}$, and $20 \mu \mathrm{g} / \mathrm{ml}$ aprotin/leupeptin/PMSF). Lysates were centrifuged at $4^{\circ} \mathrm{C}$, $13,000 \times g$ for $30 \mathrm{~min}$, and the supernatants were collected. Then, $30 \mu \mathrm{g}$ of each sample was resolved by SDS-PAGE, and proteins were transferred to nitrocellulose membranes. The membranes were washed with TBST and blocked with 5\% non-fat dry milk or $3 \% \mathrm{BSA}$ and then incubated overnight at $4^{\circ} \mathrm{C}$ with each indicated primary $\mathrm{Ab}$ and HRP-conjugated secondary Ab. Signal was detected using ECL Plus chemiluminescent detection system (Amersham).

\section{Statistical Analysis}

Results were tested for statistical significance using Student's $t$-test and presented as means $\pm S D$. Before Student's $t$-test, normal distribution of the data were analyzed. For groups that were not normally distributed, Mann-Whitney tests were used. Significance was determined as ${ }^{*} p<0.05,{ }^{* *} p<0.01$, $* * * p<0.001$.

\section{RESULTS}

\section{CXCR4 Is Required for ALL Cell Migration Toward CXCL12}

It has been demonstrated that CXCR4 is relative to infiltration of T-ALL cells $(4-8,18)$; meanwhile, CXCR4 cognate ligand $\mathrm{CXCL} 12$ is a chemokine that induces cell
WB

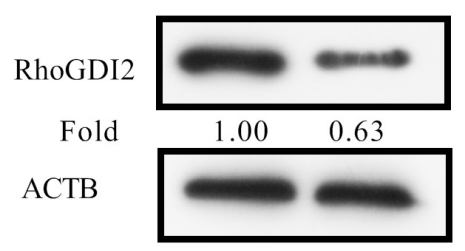

control RhoGDI2 shRNA2
B

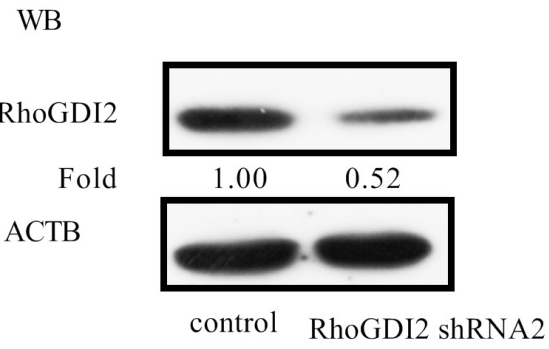

D

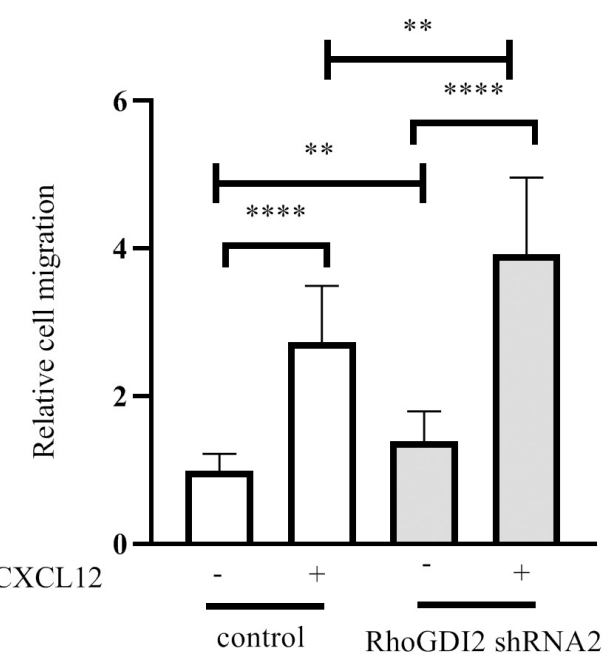

FIGURE 2 | RhoGDI2 negatively regulates T-ALL cell migration toward CXCL12. (A,B) shRNA to RhoGDI2 or control shRNA was transfected into HEK293T to generate lentivirus, JURKAT or CCRF-CEM cells were infected, and the interference efficiency was detected by Western blot, using RhoGDI2 antibody. (C,D) shRNA that successfully downregulated RhoGDI2 was used in the transwell assay. Each experiment was repeated at least three times. Western blot bands were analyzed using Image Pro Plus 6.0, and fold changes of RhoGDI2 were normalized according to ACTB. ACTB was used as the loading controls. ${ }^{*} p<0.05,{ }^{* *} p<0.01$, ${ }^{\star \star \star} p<0.001,{ }^{\star \star \star \star} p<0.0001$. 
migration (19). To identify the role of CXCR4 in T-ALL cell migration, JURKAT and CCRF-CEM ALL cell lines were pre-incubated with or without the antagonist of CXCR4, AMD3100 in each transwell assay. The results show that AMD3100 inhibits cell migration toward CXCL12 (Figures 1A,B). After CXCR4 in JURKAT and CCRF-CEM cells was reduced by shRNAs (Figures 1C,D), another transwell assay was conducted. The results show that CXCR4 shRNAs significantly reduce cell migration toward CXCL12
(Figures 1E,F). Therefore, CXCR4 is required for T-ALL cell migration toward CXCL12.

\section{RhoGDI2 Inhibits CXCR4-Mediated}

\section{T-ALL Cell Migration Toward CXCL12}

RhoGDI2 is reported to negatively or positively regulate RhoGTPases (20). We previously demonstrated that RhoA and RhoC were required for JURKAT migration toward
A
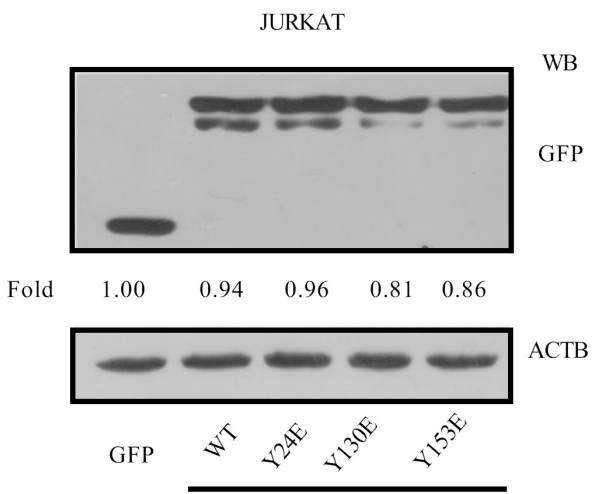

GFP-RhoGDI2

C
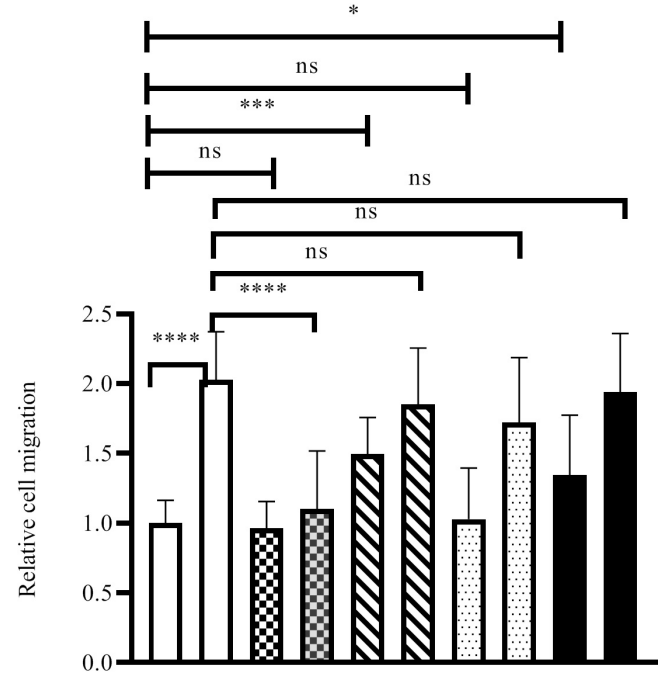

CXCL12
B

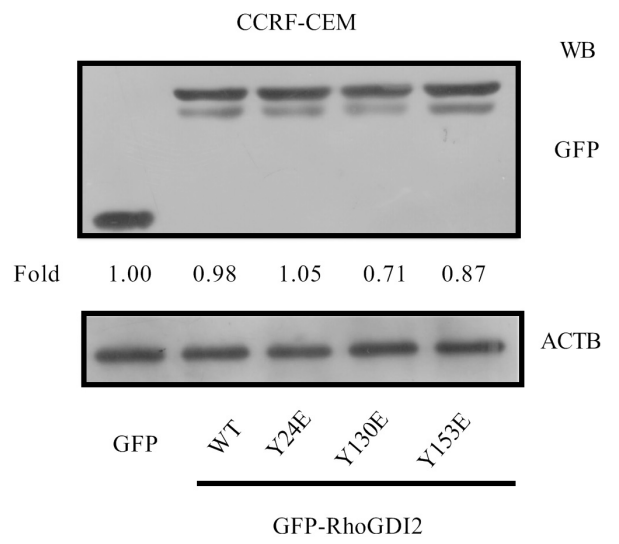

D

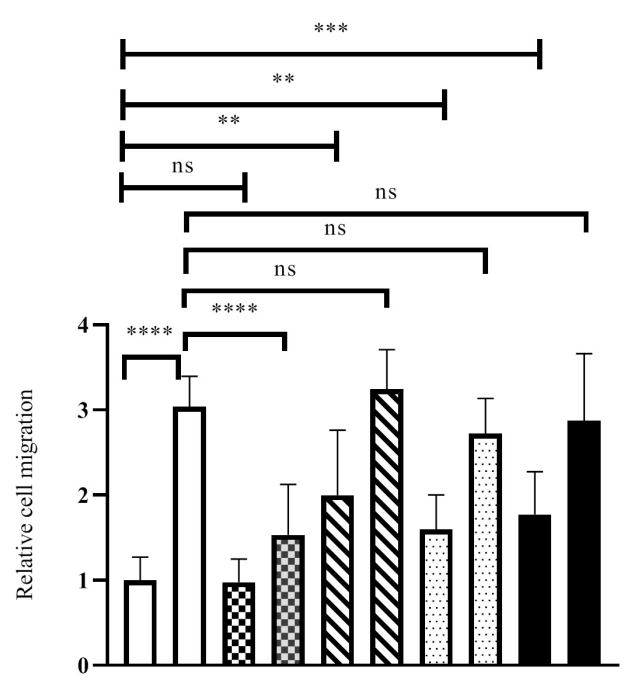

CXCL12
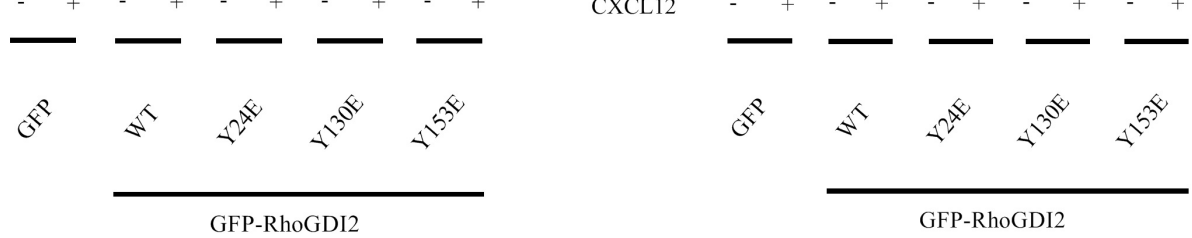

GFP-RhoGDI2

FIGURE 3 | Phosphorylation of RhoGDI2 Tyr 24, 130, or 153 recovered CXCL12-mediated ALL migration. JURKAT (A) or CCRF-CEM (B) cells were infected with lentivirus containing nucleotides expressing GFP-RhoGDI2-WT, Y24E, Y130E, or Y153E, and the overexpression levels of WT or mutated RhoGDI2 were detected $48 \mathrm{~h}$ after infection by Western blot, using GFP antibody. (C,D) Transfected cells were used in the transwell assay. Each experiment was repeated at least three times. Western blot bands were analyzed using Image Pro Plus 6.0, and fold changes of GFP, GFP-RhoGDI2-WT, or mutants were normalized according to ACTB. ACTB was used as the loading controls. ${ }^{\star} p<0.05,{ }^{* \star} p<0.01,{ }^{* \star *} p<0.001,{ }^{* \star \star *} p<0.0001$. 
A

JURKAT

WB
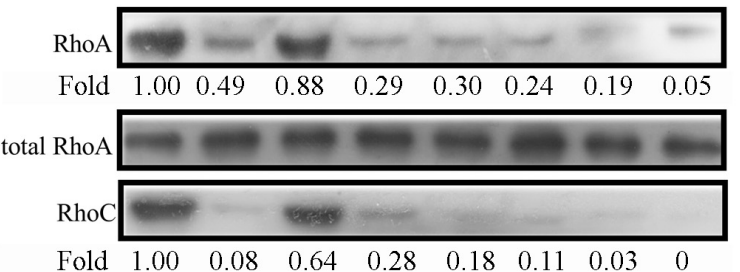

total Rho

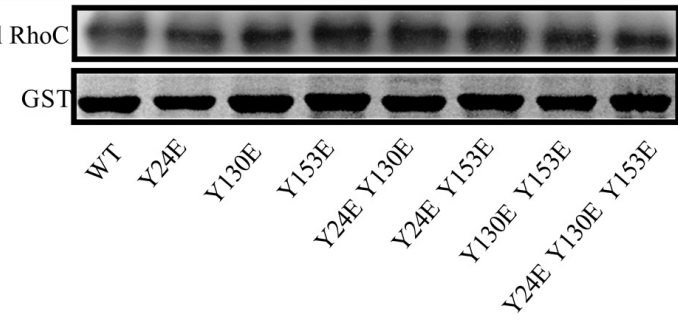

GST-RhoGDI2
B

WB

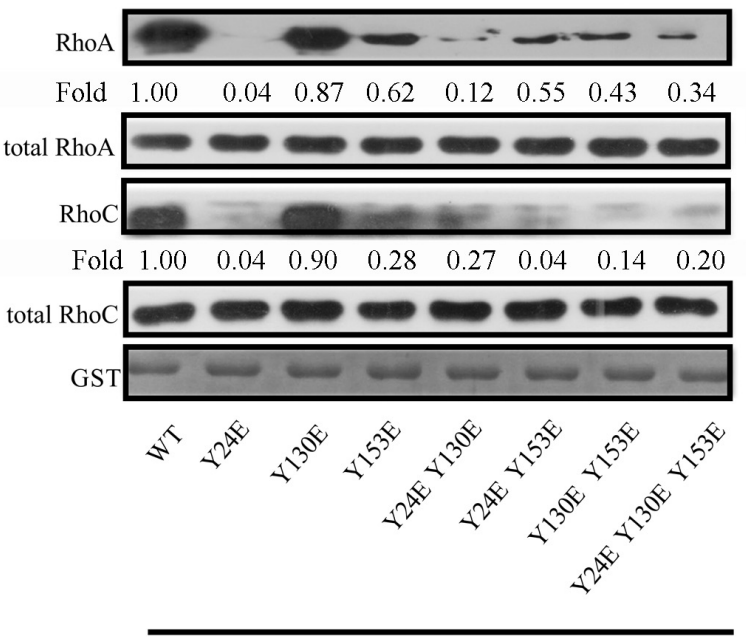

GST-RhoGDI2

FIGURE 4 | Phosphorylation of Tyr24 or Tyr153 dissociates RhoA or RhoC from RhoGDI2 and accelerates the activation of RhoA and RhoC. Cell lysates of JURKAT (A) or CCRF-CEM (B) cells were incubated with WT or mutants of GST-GDI2 in the GST pull-down assay. Then, RhoA or RhoC antibody was used in the Western blot assay to detect the level of RhoA or RhoC bound in the complex. Western blot bands were analyzed using Image Pro Plus 6.0, and fold changes of bound RhoA or RhoC were normalized according to total RhoA or RhoC. Total RhoA or RhoC was used as loading controls. GST-RhoGDI2-WT or mutants equal to that used in the GST pull-down assay were loaded and stained with coomassie blue and used as another control.

CXCL12 (21). Therefore, we hypothesized that RhoGDI2 may play a critical role in CXCR4-mediated T-ALL cell migration. To identify the role of RhoGDI2 in the migration mediated by CXCR4, shRNAs to RhoGDI2 were generated and transfected into JURKAT or CCRF-CEM cells. Results show that RhoGDI2 sh2 significantly reduces the amount of RhoGDI2 in both cell lines (Figures 2A,B). Therefore, RhoGDI2 sh2 was used in the following transwell assay. Results show that RhoGDI2 sh2 significantly reduces the cell number migrated to CXCL12, indicating that RhoGDI2 inhibits CXCR4-mediated T-ALL migration toward CXCL12 (Figures 2C,D).

\section{Phosphorylation of RhoGDI2 on Y24 and Y153 Negatively Regulates Its Inhibition to CXCR4-Mediated ALL Migration by Releasing RhoA and RhoC From RhoGDI2}

Previous studies report tyrosine-phosphorylation of RhoGDI2 on Y24, Y130, and Y153 (16, 22). To evaluate the effects of RhoGDI2 phosphorylation on RhoA, RhoC, and CXCL12-induced ALL migration, Y24, Y130, or Y153 were mutated into Glu (E) to mimic the phosphorylation station following relevant reference (23). Results show that phosphorylation of RhoGDI2 on Y24, Y130, or Y153 recovered CXCL12-mediated ALL migration (Figures 3A-D).
Phosphorylation of RhoGDI has been reported to stabilize the RhoA-RhoGDI complex in neutrophil cytosol (24). GST pulldown results show that RhoGDI2 24E or RhoGDI2 153E bind less RhoA and RhoC than RhoGDI2 130E does; however, they all bound less RhoA and RhoC than wild-type RhoGDI2 does (Figures 4A,B), suggesting that phosphorylation of Y24 or Y153 dissociates RhoA or RhoC from RhoGDI2 and accelerates the activation of RhoA and RhoC although the phosphorylation of Y130 has less effects on RhoA or RhoC activation.

\section{Src Is Activated by CXCL12 and Responsible for the Phosphorylation of RhoGDI2 on Y153}

It has been previously reported that the Src family kinase was activated and plays a role in tyrosine-phosphorylation in migration cell migration (25). CXCL12 induces migration via Src-mediated CXCR4-EGFR cross-talk in gastric cancer cells (26). Therefore, we detected the activation of Src in CXCL12treated JURKAT and CCRF-CEM cells. The results show that Src is activated in both cell lines (Figures 5A,B), providing a prerequisite for phosphorylation of RhoGDI2. To identify which tyrosine amino acid is phosphorylated by Src, active Src and specific mutants were used in the in vitro kinase assay. Results show that Src is critical for the phosphorylation of RhoGDI2 on Y153 (Figure 5C).

Furthermore, siRNA to Src was transfected into JURKAT and CCRF-CEM cells. Results show that migration is inhibited by 


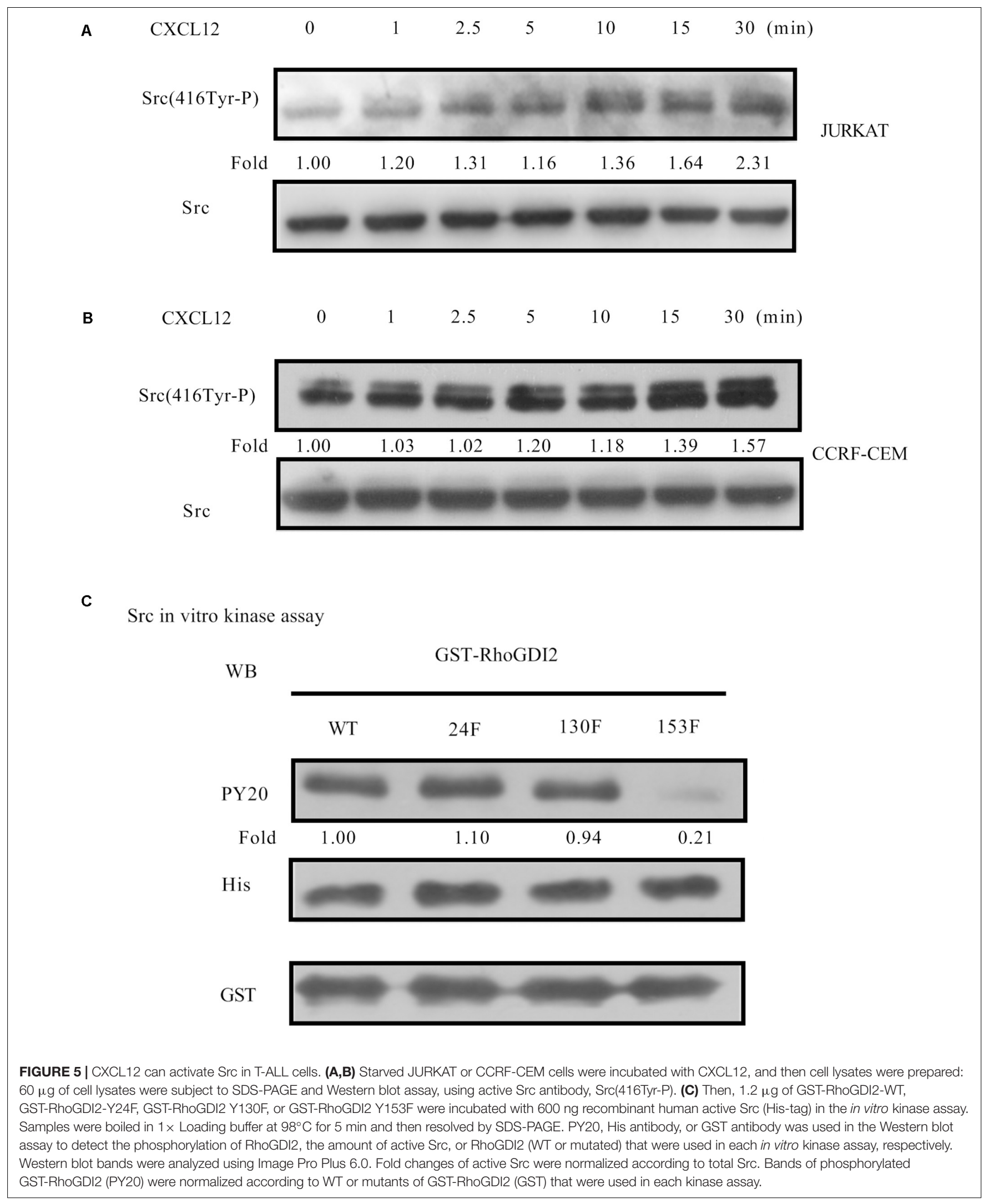


A

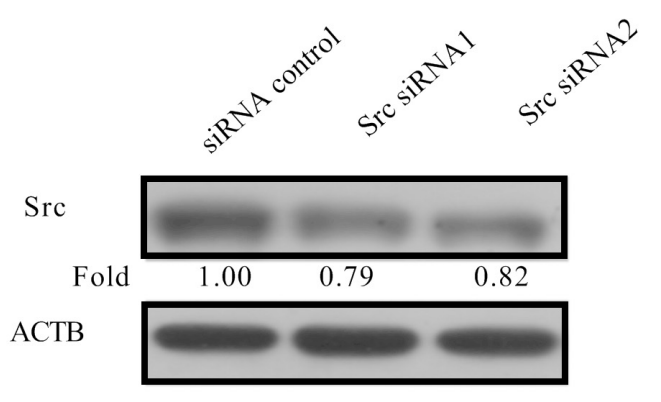

C

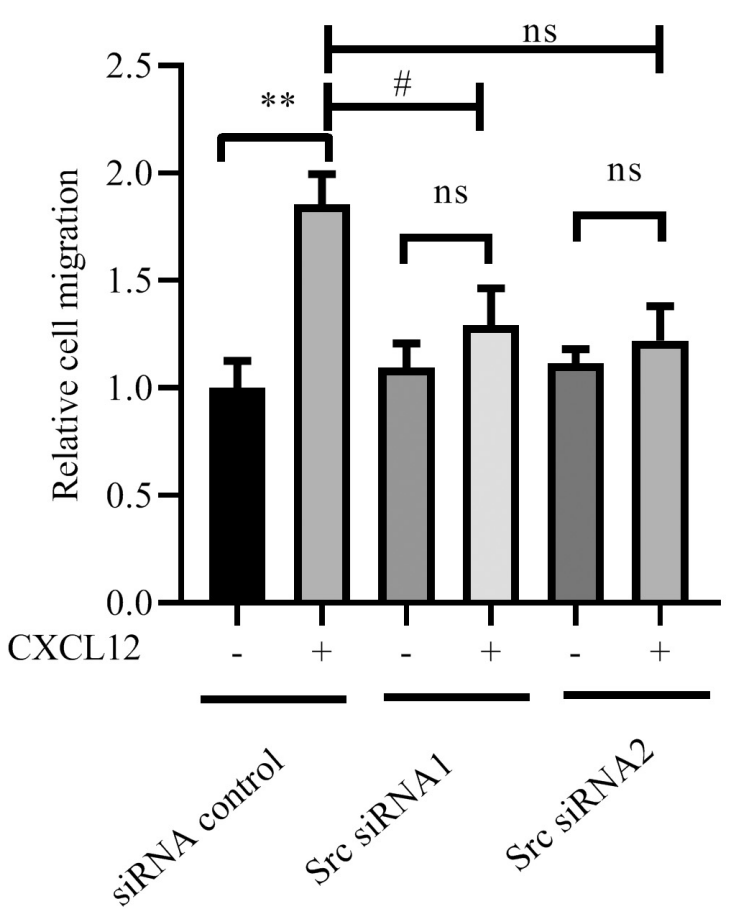

B

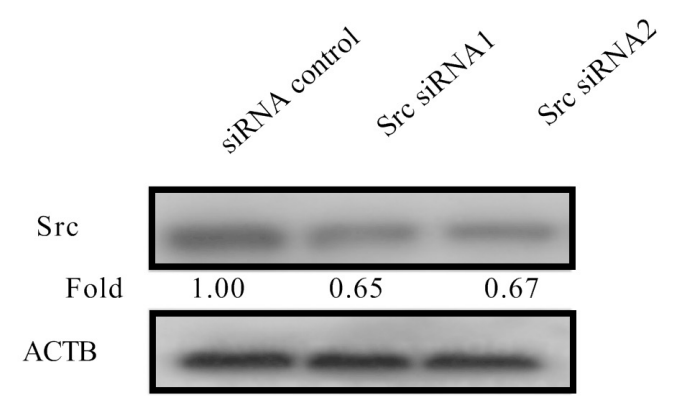

D

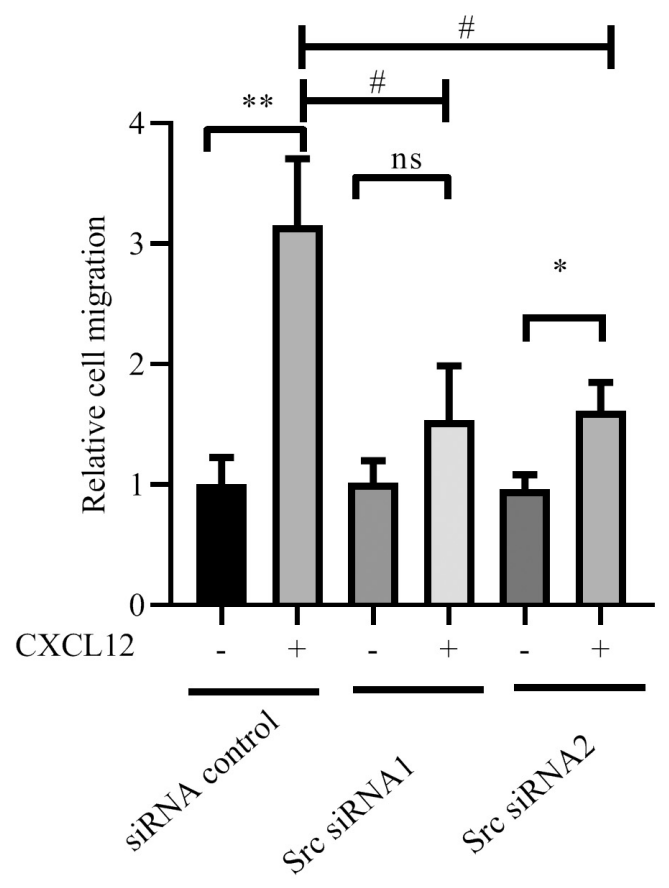

FIGURE 6 | Knockdown expression of Src by siRNAs inhibited CXCR4-mediated T-ALL migration toward CXCL12. (A,B) siRNAs were transfected into JURKAT or CCRF-CEM cells using the methods described in the materials and methods section; $48 \mathrm{~h}$ after transfection, cell lysates were prepared for Western blot assay to detect the interference efficiency of siRNAs on Src. (C,D) Transfected cells were used in the transwell assay. Each experiment was repeated at least three times. Western blot bands were analyzed using Image Pro Plus 6.0, and fold changes of Src were normalized according to ACTB. ACTB was used as the loading controls. ${ }^{\star} p<0.05,{ }^{\star \star} p<0.01$, comparing with the negative control (CXCL12-) group. ${ }^{\#} p<0.05$ comparing with the positive control (CXCL12+) group.

siRNA (Figures 6A-D), demonstrating that Src is required for CXCR4-mediated leukemia cell migration toward CXCL12.

\section{ABL1 Is Activated by CXCL12 and Responsible for the Phosphorylation of RhoGDI2 on Y24 and Y130}

GPS 5.0 predicted that Y24 may be phospholated by ABL1 (27), and KinasePhos prediction results show that Y130 may be phosphorylated by ABL1 (28). Therefore, we tested the activation of ABL1 in the CXCR4 signaling pathway and found that ABL1 is activated in response to CXCL12 (Figures 7A,B). Active ABL1 can phosphorylate RhoGDI2 on Y24 and Y130 (Figure 7C).

Furthermore, Src family kinase Lck is absolutely required for the proliferation and survival of T-ALL cells that depend on NUP214-ABL1 activity (29). Therefore, activation of Lck was detected after cells were stimulated with CXCL12. Results show that Lck is activated by CXCL12 (Figures 8A,B). The migration of T-ALL cells mediated by CXCR4 is inhibited when siRNAs to 
A

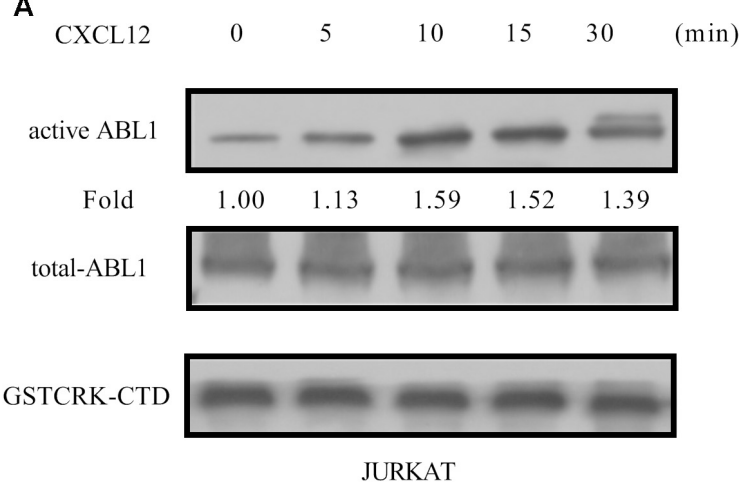

B

$\begin{array}{lllllll}\text { CXCL12 } & 0 & 5 & 10 & 15 & 30 & (\mathrm{~min})\end{array}$

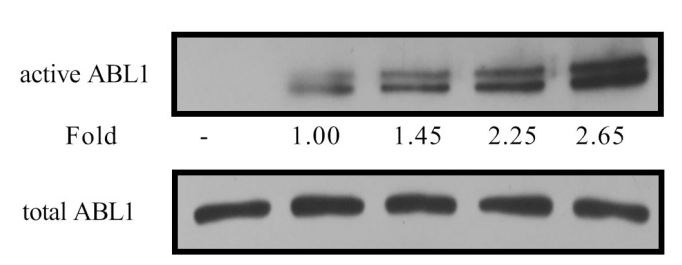

GST-CRK-CTD

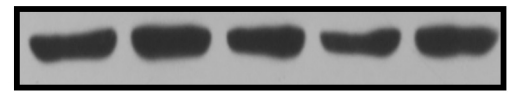

CCRF-CEM

C

CXCL12

ABL1
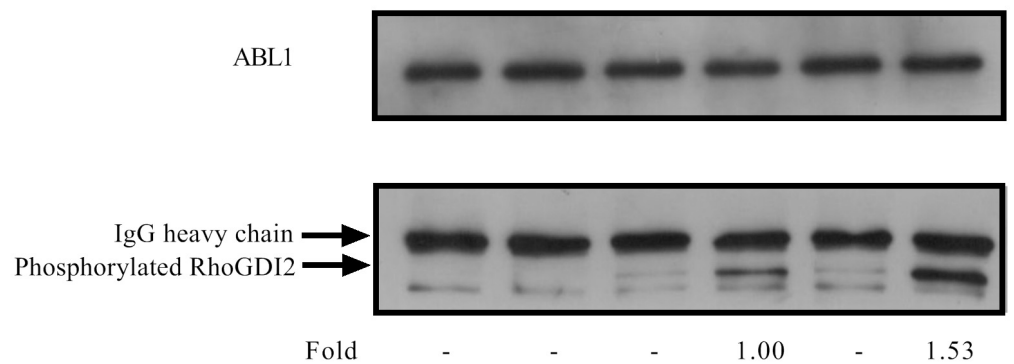

GST-RhoGDI2

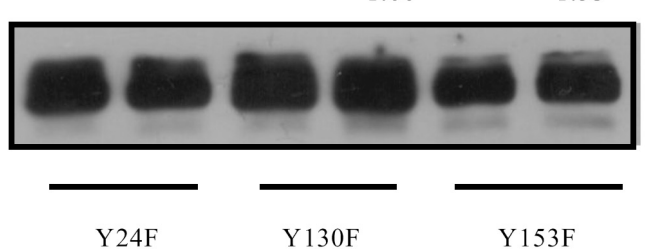

FIGURE 7 | CXCL12 can activate ABL1 in T-ALL cells. (A,B) Starved JURKAT or CCRF-CEM cells were incubated with CXCL12, and then cell lysates were prepared: $500 \mu \mathrm{g}$ of cell lysates were incubated with GST-Crk-CTD in the GST pull-down assay, followed by Western blot assay, using the antibody to ABL1. Western blot bands were analyzed using Image Pro Plus 6.0, and fold changes of active ABL1 were normalized according to GST-CRK-CTD. Total ABL1 was used as another loading control. (C) Next, $2 \mu \mathrm{g}$ of GST-RhoGDI2-Y24F, GST-RhoGDI2 Y130F, or GST-RhoGDI2 Y153F were introduced and incubated with active ABL1 immunoprecipitated from CCRF-CEM cells treated with CXCL12 at $37^{\circ} \mathrm{C}$ for $30 \mathrm{~min}$ in the in vitro kinase buffer for 30 min. Samples were boiled in $1 \times$ Loading buffer at $98^{\circ} \mathrm{C}$ for $5 \mathrm{~min}$ and then resolved by SDS-PAGE. PY20, ABL1, or GST antibody was used in the Western blot assay to detect the phosphorylation of RhoGDI2, the amount of active ABL1, or RhoGDI2 (WT or mutated) that we used in each in vitro kinase assay, respectively. Phosphorylated RhoGDI2 were normalized according to the mutants of GST-RhoGDI2. ABL1 was used as another loading control.

Lck, whose sequences are shown in Table 1, were transfected into JURKAT or CCRF-CEM cells (Figures 8C,D).

Finally, siRNA to ABL1 was transfected into JURKAT and CCRF-CEM cells. Results show that migration is inhibited by siRNA (Figures 9A-D), demonstrating that ABL1 is required for CXCR4-mediated leukemia cell migration toward CXCL12.

Taken together, RhoGDI2 is a critical signal molecule downstream of CXCR4. CXCL12/CXCR4 triggers the activation of downstream kinases, including Src and ABL1 responsible for the phosphorylation of RhoGDI2 on Y153, Y24, and Y130. Phosphorylation of RhoGDI2 on Y24 and Y153 releases RhoA and RhoC from RhoGDI2, which recovers CXCR4-mediated migration toward CXCL12 although the phosphorylation of
Y130 has less effect on RhoA or RhoC binding. Therefore, RhoGDI2 may be a brake for CXCR4-positive T-ALL migration (Figure 10).

\section{DISCUSSION}

Xenotransplantation of NSG mice with Jurkat or CCRF-CEM cells demonstrates that both can infiltrate the CNS at similar proportions and that antagonizing CXCR4 prevents the clinical features of the disease and T-ALL cell CNS tropism (4). Pediatric acute lymphoid leukemia-phase II (TARGET, 2018) data from cBioPortal for cancer genomics $(30,31)$ reveals that 
A
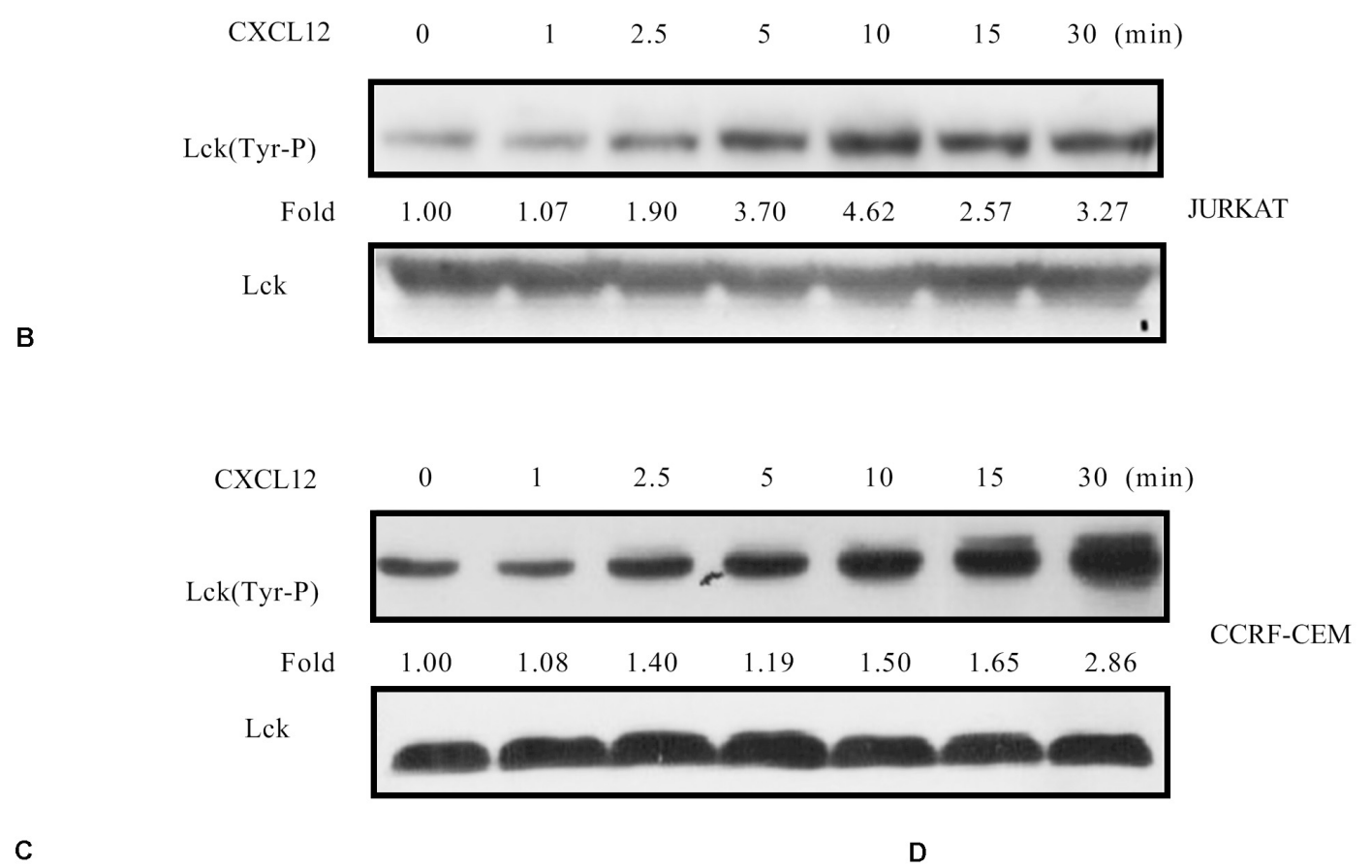

C

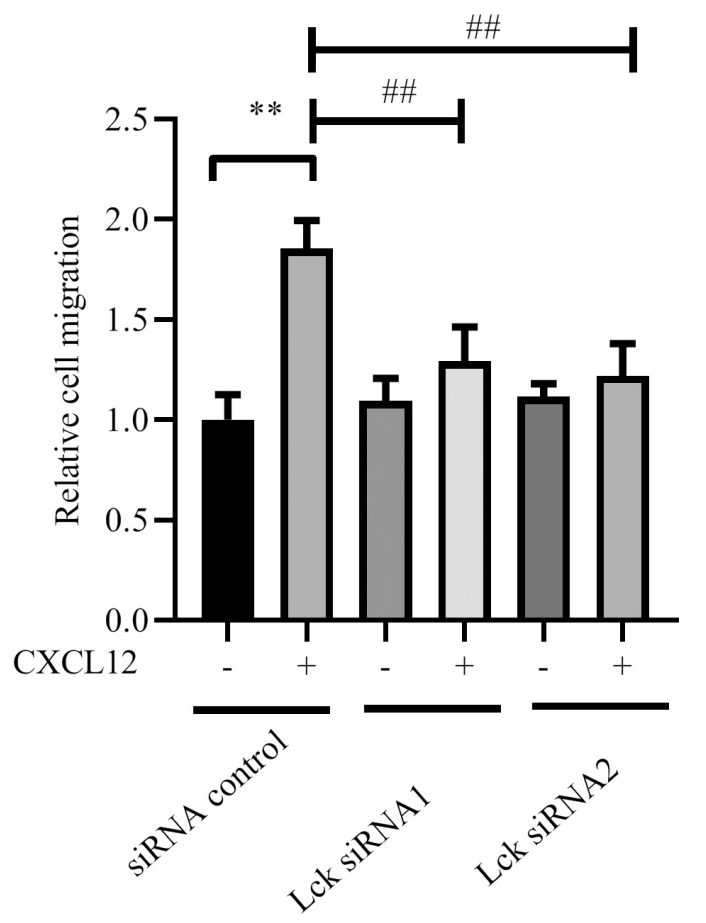


A

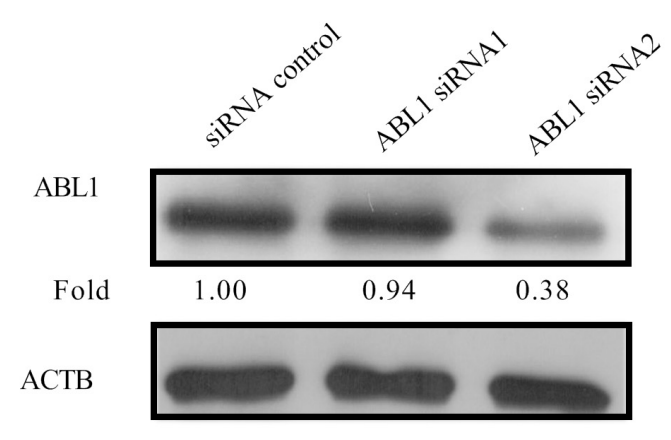

C

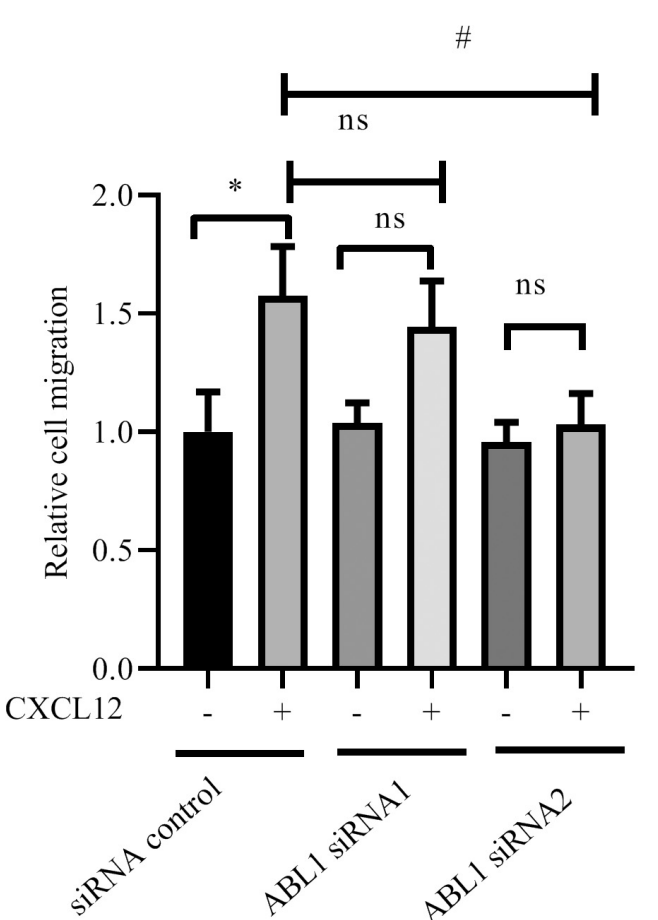

B

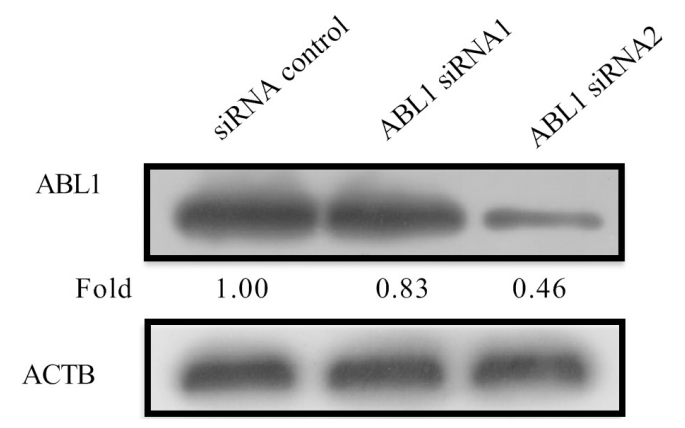

D

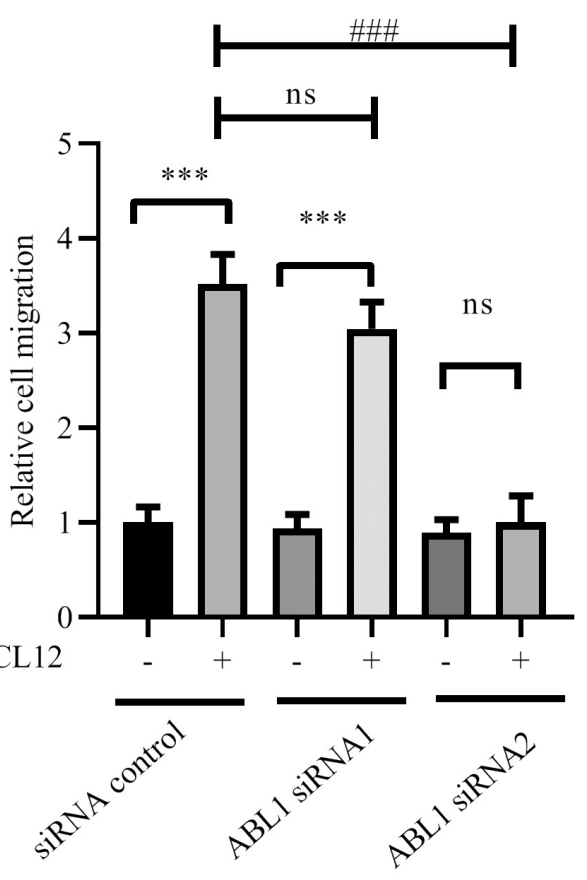

FIGURE 9 | Knockdown expression of ABL1 by siRNAs inhibited CXCR4-mediated T-ALL migration toward CXCL12. (A,B) siRNAs were transfected into JURKAT or CCRF-CEM cells using the methods described in the materials and methods section; $48 \mathrm{~h}$ after transfection, cell lysates were prepared for Western blot assay to detect the interference efficiency of siRNAs on ABL1. Western blot bands were analyzed using Image Pro Plus 6.0, and fold changes of ABL1 were normalized according the loading control ACTB. (C,D) Transfected cells were used in the transwell assay. Each experiment was repeated at least three times. ${ }^{\star} p<0.05$, ${ }^{\star \star *} p<0.001$, comparing with the negative control (CXCL12-) group. ${ }^{\#} p<0.05,{ }^{\# \# \#} p<0.001$, comparing with the positive control (CXCL12+) group.

RhoGDI2 gene ARHGDIB deep depletion is closely relative to the bone marrow site of relapse in B-ALL. However, due to the restriction of the database source, the role of RhoGDI2 in T-ALL progression remains to be investigated. Here, we find that RhoGDI2 is phosphorylated by Src and ABL1 in response to CXCR4 stimulation by CXCL12 in T-ALL cell lines and that RhoGDI2 decreases the CXCR4-mediated T-ALL cell line migration toward CXCL12 (Figures 1, 2). Because migration is necessary for infiltration and consequent relapse of leukemia, it would be interesting to explore whether RhoGDI2 brakes CXCR4-mediated T-ALL infiltration in vivo.

Recent reports show that RhoGDI2 participates in $\beta 1$ insideout signaling (16) and $\beta 2$ outside-in signaling (20) in $\mathrm{T}$ lymphocytes. Phosphorylations of p85-bound RhoGDI2 on Y130 and Y153 by $\mathrm{c}-\mathrm{Abl}$ and $\mathrm{Src}$ are required for the complex to be recruited to PSGL1 and thereby regulates $\beta 1$ integrinmediated T cell adhesion to VCAM-1 (16). Upon P-selectin glycoprotein ligand-1 engagement, RhoGDI2 is phosphorylated at Y24 and Y153 by Src, ABL1, and Syk (20). In bladder 


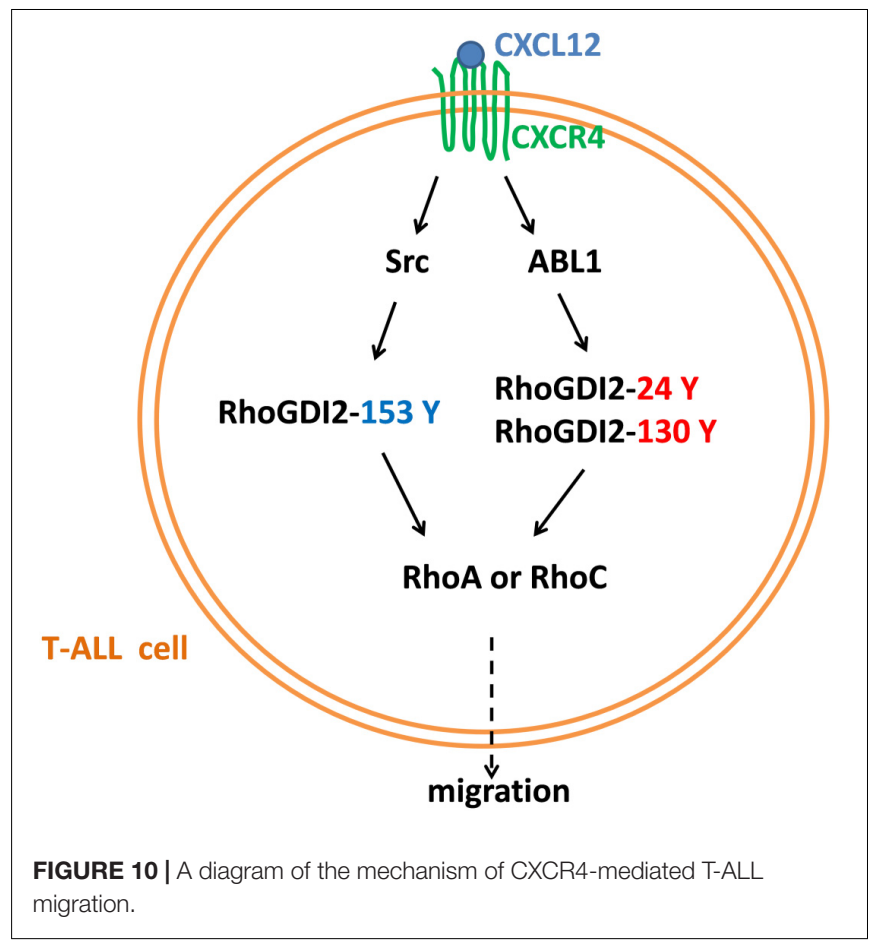

cancer, it was demonstrated that, except Y153, Y24 was also the primary Src phosphorylation site (22). The Y24 mutant also showed a partial reduction in phosphorylation by Src, which was significant in HEK293T but noticeably less in UMUC3 human bladder cancer cells. Src phosphorylation in RhoGDI2 has been involved in migration of neutrophils toward fMLP (24). In our in vitro kinase assay, results show that active Src primarily activates Y153 rather than Y24 of RhoGDI2 (Figure 5C).

Proteomic analyses have identified phosphorylation in Y24 of RhoGDI2 in pervanadate-treated JURAT cells, an established leukemic T-cell line $(32,33)$. Using JURKAT and CCRFCEM T-ALL cell lines, we find that RhoGDI2 Y24 and Y130 are phosphorylated by ABL1 (Figure 7C). Although ABL1 is reported to phosphorylate Y130 of p85-bound RhoGDI2 upon PSGL-1 ligation (16), we haven't found any scholarly report that ABL1 phosphorylation in Y24 and Y130 of RhoGDI2 is involved in the migration of T-ALL cells toward CXCL12, suggesting the involvement of ABL1 in T-ALL. Consistently, the NUP214ABL1 fusion protein, which is a constitutively active form of ABL1, is found in $6 \%$ of patients with T-ALL and promotes proliferation and survival of T-lymphoblasts (34). Here, we found that Src and ABL1 are involved in the migration of the T-ALL cell lines that is mediated by CXCR4 (Figures 6, 9). These findings underscore the potential of Src kinase inhibitors and of the dual ABL1/Src kinase inhibitors dasatinib and bosutinib for the treatment of T-ALL. Additionally, knockdown of PRL3, highly expressed in T-ALL patients, significantly impedes T-ALL cell migration capacity in vitro and in vivo (35). The Src signaling pathway is affected by PRL-3 although Src is not a direct substrate of PRL-3 (35). Coincidentally, in a mass spectrometry experiment to identify protein interaction partners of ABL1, we found that RPL-3 was in the immunoprecipitation complex of ABL1 (unpublished data). We speculate that ABL1 may be a cooperator of RPL-3 to regulate Src. However, this hypothesis needs further demonstration.

RhoGDI1 Tyr156 is the major site of Src phosphorylation, whose phosphorylation decreases its affinity for RhoGTPase, including Rac1, Cdc42, and RhoA (23). Phosphorylation on Tyr156 in RhoGDI abrogates the ability of RhoGDI1 to rebind and inhibit activated Rho GTPase both in vitro and in intact cells (23). Tyrosine-phosphorylated RhoGDI1 localizes to areas of membrane ruffling and to podosome rosettes in Src-transformed NIH-3T3 fibroblasts (23). Phosphorylation of Rho GDI1 stabilizes the RhoA-RhoGDI1 complex in neutrophil cytosol (24). RhoGDI2 contains an equivalent tyrosine residue at aa 153. Phosphorylation decreases the amount of Rac1 in RhoGDI2 complexes and increases RhoGDI2 association with cell membranes (22). Although it has been reported that RhoGDI2 positively regulates the activation of Rac1 and Cdc42 (20), we find the phosphorylation on 24 and 153 tyrosine complex less RhoA or RhoC in human T-ALL cell lines (Figure 3), raising a possibility to release the negative regulatory role of RhoGDI2 in T-ALL cell migration toward CXCL12 (Figure 4).

\section{DATA AVAILABILITY STATEMENT}

The raw data supporting the conclusions of this article will be made available by the authors, without undue reservation.

\section{AUTHOR CONTRIBUTIONS}

JL and JW designed the study, collated the data, and carried out data analyses. JW and HZ conducted the experiments. JL produced the initial draft of the manuscript. JW and LW contributed to drafting the manuscript. All authors have read and approved the final submitted manuscript.

\section{FUNDING}

This work was supported by the National Natural Science Foundation of China (grant no. 31401216), Research Project Supported by the Shanxi Scholarship Council of China (grant no. 2017-012), and the Natural Science Foundation of Shanxi Province of China (grant no. 201901D111010).

\section{ACKNOWLEDGMENTS}

GFP-tagged WT human RhoGDI2 in lentiviral pWPXLd vector and shRNAs targeting RhoGDI2 or random control in lentiviral pDSL_hpUGIP vector were kindly provided by Professor Xianlu Zeng from Northeast Normal University, Changchun, China. We wish to thank Prof. M. James C. Crabbe in Wolfson College, University of Oxford, for suggestions and revision of the manuscript. 


\section{REFERENCES}

1. Wu C, Li W. Genomics and pharmacogenomics of pediatric acute lymphoblastic leukemia. Crit Rev Oncol Hematol. (2018) 126:100-11. doi: 10.1016/j.critrevonc.2018.04.002

2. Inaba H, Greaves M, Mullighan CG. Acute lymphoblastic leukaemia. Lancet. (2013) 381:1943-55. doi: 10.1016/s0140-6736(12)62187-4

3. Ko RH, Ji L, Barnette P, Bostrom B, Hutchinson R, Raetz E, et al. Outcome of patients treated for relapsed or refractory acute lymphoblastic leukemia: a therapeutic advances in childhood leukemia consortium study. J Clin Oncol. (2010) 28:648-54. doi: 10.1200/jco.2009.22.2950

4. Jost TR, Borga C, Radaelli E, Romagnani A, Perruzza L, Omodho L, et al. Role of CXCR4-mediated bone marrow colonization in CNS infiltration by T cell acute lymphoblastic leukemia. J Leukoc Biol. (2016) 99:1077-87. doi: 10.1189/jlb.5MA0915-394R

5. Matsumoto T, Jimi S, Hara S, Takamatsu Y, Suzumiya J, Tamura K. Am80 inhibits stromal cell-derived factor-1-induced chemotaxis in T-cell acute lymphoblastic leukemia cells. Leuk Lymphoma. (2010) 51:507-14. doi: 10. 3109/10428190903560180

6. Crazzolara R, Kreczy A, Mann G, Heitger A, Eibl G, Fink FM, et al. High expression of the chemokine receptor CXCR4 predicts extramedullary organ infiltration in childhood acute lymphoblastic leukaemia. Br J Haematol. (2001) 115:545-53. doi: 10.1046/j.1365-2141.2001.03164.x

7. Alsadeq A, Fedders H, Vokuhl C, Belau NM, Zimmermann M, Wirbelauer T, et al. The role of ZAP70 kinase in acute lymphoblastic leukemia infiltration into the central nervous system. Haematologica. (2017) 102:346-55. doi: 10. 3324/haematol.2016.147744

8. Annels NE, Willemze AJ, van der Velden VH, Faaij CM, van Wering E, SieGo DM, et al. Possible link between unique chemokine and homing receptor expression at diagnosis and relapse location in a patient with childhood T-ALL. Blood. (2004) 103:2806-8. doi: 10.1182/blood-2003-06-1812

9. Vadillo E, Dorantes-Acosta E, Pelayo R, Schnoor M. T cell acute lymphoblastic leukemia (T-ALL): New insights into the cellular origins and infiltration mechanisms common and unique among hematologic malignancies. Blood Rev. (2018) 32:36-51. doi: 10.1016/j.blre.2017.08.006

10. Scherle P, Behrens T, Staudt LM. Ly-GDI, a GDP-dissociation inhibitor of the RhoA GTP-binding protein, is expressed preferentially in lymphocytes. Proc Natl Acad Sci USA. (1993) 90:7568-72. doi: 10.1073/pnas.90.16.7568

11. Boulter E, Garcia-Mata R, Guilluy C, Dubash A, Rossi G, Brennwald PJ, et al. Regulation of Rho GTPase crosstalk, degradation and activity by RhoGDI1. Nat Cell Biol. (2010) 12:477-83. doi: 10.1038/ncb2049

12. Adra CN, Ko J, Leonard D, Wirth LJ, Cerione RA, Lim B. Identification of a novel protein with GDP dissociation inhibitor activity for the ras-like proteins CDC42Hs and rac I. Genes Chromosomes Cancer. (1993) 8:253-61. doi: $10.1002 /$ gcc. 2870080408

13. Scheffzek K, Stephan I, Jensen ON, Illenberger D, Gierschik P. The RacRhoGDI complex and the structural basis for the regulation of Rho proteins by RhoGDI. Nat Struct Biol. (2000) 7:122-6. doi: 10.1038/72392

14. Yin L, Schwartzberg P, Scharton-Kersten TM, Staudt L, Lenardo M. Immune responses in mice deficient in Ly-GDI, a lymphoid-specific regulator of Rho GTPases. Mol Immunol. (1997) 34:481-91. doi: 10.1016/s0161-5890(97) 00053-9

15. Leffers H, Nielsen MS, Andersen AH, Honore B, Madsen P, Vandekerckhove J, et al. Identification of two human Rho GDP dissociation inhibitor proteins whose overexpression leads to disruption of the actin cytoskeleton. Exp Cell Res. (1993) 209:165-74. doi: 10.1006/excr.1993.1298

16. Luo J, Xu T, Li C, Ba X, Wang X, Jiang Y, et al. p85-RhoGDI2, a novel complex, is required for PSGL-1-induced betal integrin-mediated lymphocyte adhesion to VCAM-1. Int J Biochem Cell Biol. (2013) 45:2764-73. doi: 10.1016/j.biocel. 2013.09.005

17. Chen C, Shang X, Xu T, Cui L, Luo J, Ba X, et al. c-Abl is required for the signaling transduction induced by L-selectin ligation. Eur J Immunol. (2007) 37:3246-58. doi: 10.1002/eji.200737221

18. Kawaguchi A, Orba Y, Kimura T, Iha H, Ogata M, Tsuji T, et al. Inhibition of the SDF-1alpha-CXCR4 axis by the CXCR4 antagonist AMD3100 suppresses the migration of cultured cells from ATL patients and murine lymphoblastoid cells from HTLV-I Tax transgenic mice. Blood. (2009) 114:2961-8. doi: 10. 1182/blood-2008-11-189308
19. Cyster JG. Chemokines and cell migration in secondary lymphoid organs. Science. (1999) 286:2098-102. doi: 10.1126/science.286.5447.2098

20. Liu W, Wang X, Wang S, Ba X, Xu T, Wang X, et al. RhoGDI2 positively regulates the Rho GTPases activation in response to the beta2 outside-in signaling in T cells adhesion and migration on ICAM-1. J Leukoc Biol. (2019) 106:431-46. doi: 10.1002/JLB.2A0718-272RR

21. Luo J, Li D, Wei D, Wang X, Wang L, Zeng X. RhoA and RhoC are involved in stromal cell-derived factor-1-induced cell migration by regulating F-actin redistribution and assembly. Mol Cell Biochem. (2017) 436:13-21. doi: 10. 1007/s11010-017-3072-3

22. Wu Y, Moissoglu K, Wang H, Wang X, Frierson HF, Schwartz MA, et al. Src phosphorylation of RhoGDI2 regulates its metastasis suppressor function. Proc Natl Acad Sci USA. (2009) 106:5807-12. doi: 10.1073/pnas.0810094106

23. DerMardirossian C, Rocklin G, Seo JY, Bokoch GM. Phosphorylation of RhoGDI by Src regulates Rho GTPase binding and cytosol-membrane cycling. Mol Biol Cell. (2006) 17:4760-8. doi: 10.1091/mbc.e06-06-0533

24. Bourmeyster N, Vignais PV. Phosphorylation of Rho GDI stabilizes the Rho A-Rho GDI complex in neutrophil cytosol. Biochem Biophys Res Commun. (1996) 218:54-60. doi: 10.1006/bbrc.1996.0011

25. Yang CY, Chang PW, Hsu WH, Chang HC, Chen CL, Lai CC. Src and SHP2 coordinately regulate the dynamics and organization of vimentin filaments during cell migration. Oncogene. (2019) 38:4075-94. doi: 10.1038/s41388-0190705-X

26. Cheng Y, Qu J, Che X, Xu L, Song N, Ma Y, et al. CXCL12/SDF-1alpha induces migration via SRC-mediated CXCR4-EGFR cross-talk in gastric cancer cells. Oncol Lett. (2017) 14:2103-10. doi: 10.3892/ol.2017.6389

27. Xue Y, Zhou F, Zhu M, Ahmed K, Chen G, Yao X. GPS: a comprehensive www server for phosphorylation sites prediction. Nucleic Acids Res. (2005) 33:W184-7. doi: 10.1093/nar/gki393

28. Yung-Hao W, Tzong-Yi L, Liang HK, Huang CM, Wang TY, Yang YH, et al. KinasePhos 2.0: a web server for identifying protein kinase-specific phosphorylation sites based on sequences and coupling patterns. Nucleic Acids Res. (2007) 35:W588-94.

29. Keersmaecker KD, Porcu M, Cox L, Girardi T, Vandepoel R, Beeck JOD, et al. NUP214-ABL1 mediated cell proliferation in T-cell acute lymphoblastic leukemia is dependent on the LCK kinase and various interacting proteins. Haematologica. (2014) 99:85-93. doi: 10.3324/haematol.2013. 088674

30. Cerami E, Gao J, Dogrusoz U, Gross BE, Schultz N. The cBio cancer genomics portal: an open platform for exploring multidimensional cancer genomics data. Cancer Discov. (2012) 2:401-4.

31. Gao J, Aksoy BA, Dogrusoz U, Dresdner G, Gross B, Sumer SO, et al. Integrative analysis of complex cancer genomics and clinical profiles using the cBioPortal. Sci Signal. (2013) 6:11-1.

32. Rush J, Moritz A, Lee KA, Guo A, Goss VL, Spek EJ, et al. Immunoaffinity profiling of tyrosine phosphorylation in cancer cells. Nat Biotechnol. (2005) 23:94-101. doi: 10.1038/nbt1046

33. Schneider U, Schwenk HU, Bornkamm G. Characterization of EBV-genome negative "null" and " $\mathrm{T}$ " cell lines derived from children with acute lymphoblastic leukemia and leukemic transformed non-Hodgkin lymphoma. Int.J.Cancer. (1977) 19:621-6.

34. De Keersmaecker K, Marynen P, Cools J. Genetic insights in the pathogenesis of T-cell acute lymphoblastic leukemia. Haematologica. (2005) 90:1116-27.

35. Wei M, Haney MG, Rivas DR, Blackburn JS. Protein tyrosine phosphatase 4A3 (PTP4A3/PRL-3) drives migration and progression of T-cell acute lymphoblastic leukemia in vitro and in vivo. Oncogenesis. (2020) 9:6. doi: 10.1038/s41389-020-0192-5

Conflict of Interest: The authors declare that the research was conducted in the absence of any commercial or financial relationships that could be construed as a potential conflict of interest.

Copyright (c) 2020 Luo, Wang, Zheng and Wang. This is an open-access article distributed under the terms of the Creative Commons Attribution License (CC BY). The use, distribution or reproduction in other forums is permitted, provided the original author(s) and the copyright owner(s) are credited and that the original publication in this journal is cited, in accordance with accepted academic practice. No use, distribution or reproduction is permitted which does not comply with these terms. 